\title{
Effect of Peroral Endoscopic Myotomy on Esoph- ageal Motor Function
}

\author{
Su Jin Hong \\ Digestive Disease Center and Research Institute, Department of Internal Medicine, Soonchunhyang University College of Medicine, Bucheon, \\ Korea
}

Article: Myotomy of distal esophagus influences proximal esophageal contraction and upper esophageal sphincter relaxation in patients with achalasia after peroral endoscopic myotomy

Ren $Y$, Tang $X$, Chen F, et al.

(J Neurogastroenterol Motil 2016;22:78-85)

Achalasia is characterized by incomplete esophagogastric junction (EGJ) relaxation and loss of peristalsis. The main treatment of achalasia has focused on reduction of functional EGJ obstruction by endoscopic or surgical methods. Peroral endoscopic myotomy (POEM) has been introduced as a novel treatment for achalasia. ${ }^{1}$ The procedure can supply an opportunity to perform longer length esophageal myotomy compared to surgical myotomy.

After treatment of achalasia, most patients show decreased EGJ integrated relaxation pressures on high-resolution manometry. ${ }^{2}$ The longitudinal muscle contraction by deglutition induces elevation of the common cavity pressure and results in pan-esophageal pressurization. ${ }^{3} \mathrm{~A}$ small number of treated patients have shown partial recovery of peristalsis or disappearance of pan-esophageal pressurization on high-resolution manometry. ${ }^{4}$

In patients with impaired lower esophageal sphincter (LES) relaxation including achalasia and EGJ outflow obstruction, abnormalities of the upper esophageal sphincter (UES) were more frequently detected. ${ }^{5}$ The majority of UES abnormalities were hypertensive. In addition, there was a significant association between the lack of treatment response and the presence of UES dysfunction. ${ }^{5}$ A suggested mechanism of hypertensive UES was avoidance of aspiration due to accumulation of food by a neural feedback mechanism. Interestingly, the UES residual pressure (UESRP) significantly decreased after treatment in achalasia patients. ${ }^{6,7}$ The UES pressure changes were mainly detected in patients with type II achalasia, which was suggestive of early stage of disease.

In the current issue of this journal, Ren et $\mathrm{al}^{8}$ studied the correlation of motility changes between distal and proximal esophagus after POEM. Although the number of patients who were enrolled in the study was relatively small, the authors observed the changes in Eckardt scores, LES tone, contractile integral of distal segment with myotomy (CI-DM), contractile integral of proximal segment without myotomy (CI-PNM), and UES relaxation pressures. The authors observed decrease in Eckardt scores, LES tone, CI-DM, CI-PNM, and UES relaxation pressures after POEM. However, UES tone and UES relaxation duration did not change. Furthermore, a positive linear correlation between CI-DM and CI-PNM changes was observed. There was a positive correlation between

Received: December 1, 2015 Revised: December 2, 2015 Accepted: December 4, 2015

() This is an Open Access article distributed under the terms of the Creative Commons Attribution Non-Commercial License (http://creativecommons. org/licenses/by-nc/4.0) which permits unrestricted non-commercial use, distribution, and reproduction in any medium, provided the original work is properly cited.

*Correspondence: Su Jin Hong, MD, PhD Digestive Disease Center and Research Institute, Department of Internal Medicine, Soonchunhyang University College of Medicine, 170 Jomaru-ro Wonmi-gu, Bucheon, Gyenggi-do 14584, Korea Tel: +82-32-621-5087, Fax: +82-32-621-5080, E-mail: sjhong@schmc.ac.kr 
the change of UES relaxation pressure and change of contractile integral of the esophageal body in this study. The above authors suggested that distal myotomy of the esophageal body affected the motility of the proximal segment and the UES. However, they did not explain the mechanism of these phenomena. In addition, they failed to observe these motility changes of the proximal segment and UES in type I achalasia. They expected that a larger population of patients with type I achalasia might show the same motility changes such as type II and III achalasia in future studies.

In a previous study, type I achalasia showed minimal to no contraction of the longitudinal muscle of the esophagus. ${ }^{9}$ Therefore, the decreased contractile integral of the esophageal body might not be easily detectable in a small-sized group of type I achalasia after POEM. In contrast, the decreased CI-PNM in this study was not a surprising result, because the common cavity pressure naturally decreased after POEM in type II achalasia. Spastic type contraction is a characteristic feature of type III achalasia. In type III achalasia, both circular and longitudinal muscles contract, but there is severe discoordination between the 2 muscle layers. ${ }^{9}$ Additional studies will prove the motility changes of proximal esophagus and UES in each subtype of achalasia after POEM.

\section{Financial support: None.}

\section{Conflicts of interest: None.}

\section{References}

1. Pasricha PJ, Hawari R, Ahmed I, et al. Submucosal endoscopic esophageal myotomy: a novel experimental approach for the treatment of achalasia. Endoscopy 2007;39:761-764.

2. Lee BH, Shim KY, Hong SJ, et al. Peroral endoscopic myotomy for treatment of achalasia: initial results of a Korean study. Clin Endosc 2013; 46:161-167.

3. Mittal RK, Hong SJ, Bhargava V. Longitudinal muscle dysfunction in achalasia esophagus and its relevance. J Neurogastroenterol Motil 2013;19:126-136.

4. Roman S, Kahrilas PJ, Mion F, et al. Partial recovery of peristalsis after myotomy for achalasia: more the rule than the exception. JAMA Surg 2013;148:157-164.

5. Chavez YH, Ciarlelio MM, Clarke JO, Nandwani M, Stein E, Roland BC. Upper esophageal sphincter abnormalities: frequent finding on highresolution esophageal manometry and associated with poorer treatment response in achalasia. J Clin Gastroenterol 2015;49:17-23.

6. Yao S, Linghu E. Peroral endoscopic myotomy can improve esophageal motility in patients with achalasia from a large sample self-control research (66 patients). PLoS One 2015;10:e125942.

7. Wauters L, Van Oudenhove L, Selleslagh M, et al. Balloon dilation of the esophago-gastric junction affects lower and upper esophageal sphincter function in achalasia. Neurogastroenterol Motil 2014;26:69-76.

8. Ren Y, Tang X, Chen F, et al. Myotomy of distal esophagus influences proximal esophageal contraction and upper esophageal sphincter relaxation in patients with achalasia after peroral endoscopic myotomy. J Neurogastroenterol Motil 2015;22:78-85.

9. Hong SJ, Bhargava V, Jiang Y, Denboer D, Mittal RK. A unique esophageal motor pattern that involves longitudinal muscles is responsible for emptying in achalasia esophagus. Gastroenterology 2010;139:102-111. 